\title{
An Assessment of the Relationship between the Maxillary Sinus Floor and the Maxillary Posterior Teeth Root Tips Using Dental Cone-beam Computerized Tomography
}

\author{
Cenk Kilic ${ }^{a}$ \\ Kivanc Kamburoglu ${ }^{\mathrm{b}}$ \\ Selcen Pehlivan Yukselc \\ Tuncer Ozen ${ }^{d}$
}

\section{ABSTRACT}

Objectives: The aim of the present study was to assess the relationship between the maxillary sinus floor and the maxillary posterior teeth root tips using dental cone-beam CT.

Methods: A total of 87 right and 89 left maxillary sinus regions from 92 patients were examined using dental cone-beam CT. Images were analyzed by a specialist in oral and maxillofacial radiology. Perpendicular lines were drawn on the cross-sectional images between the deepest point of the maxillary sinus floor and the root tips of the maxillary first and second premolars and first, second and third molars, and the distances were measured using built-in measurement tools. Means, standard deviations and minimum and maximum values were calculated for all right and left premolars and molars. T-tests were used to compare measurements between left and right sides and between female and male patients.

Results: The distance between sinus floor and root tip was longest for the first premolar root tip and shortest for the second molar buccodistal root tip for both right and left sides. No statistically significant differences were found between the right and left side measurements or between female and male patients ( $P>$.05).

Conclusions: Knowledge of the anatomical relationship between the maxillary sinus floor and the maxillary posterior teeth root tips is important for the preoperative treatment planning of maxillary posterior teeth. (Eur J Dent 2010;4:462-467)

Key words: Maxillary sinus; Posterior teeth; Cone beam; Computerized tomography.

Department of Anatomy, Faculty of Medicine, Gulhane Military Medical Academy, Ankara, Turkey.

b Department of Oral Diagnosis and Radiology, Faculty of Dentistry, Ankara University, Ankara, Turkey.

Department of Biostatistics, Faculty of Medicine, Ankara University, Ankara, Turkey.

d Department of Oral Diagnosis and Radiology, Dental Science Center, Gulhane Military

Medical Academy, Ankara, Turkey.

\footnotetext{
Corresponding author: Cenk Kilic

Gulhane Military Medical Academy (GATA),

Faculty of Medicine, Department of

Anatomy, 06018, Etlik, Ankara, Turkey.

Phone: +903123043508

Fax: +903123810602

E-mail: ckilicmdayahoo.com
} 


\section{INTRODUCTION}

The maxillary sinus is the first of the paranasal sinuses to develop, and its growth ends with the eruption of the third molars at approximately 20 years of age. ${ }^{1}$ The inferior sinus wall is a curved structure formed by the lower third of the medial wall and the buccoalveolar wall, ${ }^{2}$ and the floor is formed by the alveolar process of the maxilla. The adult sinus is variable in its extension. In about half of the population, ${ }^{3}$ the sinus floor extends between adjacent teeth or individual roots, creating elevations in the antral surface, commonly referred to as 'hillocks'. ${ }^{4}$ The roots of the maxillary premolar, molar and occasionally canine teeth may project into the maxillary sinus. ${ }^{5}$ Because of the implications this can have on surgical procedures, it is essential for clinicians to be aware of the exact relationship between the apical roots of the maxillary teeth and the maxillary sinus floor.

Wehrbein and Diedrich ${ }^{6}$ described a positive correlation between the length of root projection into the maxillary sinus as observed on panoramic radiographs and the amount of pneumatization that occurs after extraction. Sinus expansion following extraction can greatly decrease the amount of bone height available for implant placement. ${ }^{1}$ A periapical or periodontal infection of the upper premolars and molars may spread beyond the confines of the supporting dental tissue into the maxillary sinus, causing sinusitis., ${ }^{3,7}$ Endodontic therapy or extraction of these teeth can result in penetration, ${ }^{9}$ oroantral fistulae or root displacement into the sinus cavity. ${ }^{10}$ The relationship between the dental roots and the inferior sinus wall is known to influence orthodontic tooth movement, ${ }^{11}$ and the intrusion or bodily movement of teeth across the sinus floor that occurs with orthodontic treatment has been shown to cause moderate apical root resorption and a high degree of tipping. ${ }^{6}$ The aim of this study was to assess the relationship between the maxillary sinus floor and the apices of the maxillary teeth roots using dental cone-beam CT.

\section{MATERIALS AND METHODS}

The study material comprised dental conebeam CT images (Imtec Imaging, Ardmore, OK, US) taken from 92 patients obtained from the archives of a private dentomaxillofacial radiology center. Of these, 50 (54.3\%) were female and 42 $(45.7 \%)$ were male, with a mean age of $38.8 \pm 15.3$ years (range: 10-66 years). In total, 87 dentulous right maxillary sinus regions and 89 dentulous left maxillary sinus regions were studied. All images were examined by an oral and maxillofacial radiology specialist. Lines were drawn on the crosssectional images between the deepest point of the maxillary sinus floor and the root tips of the maxillary first and second premolars and first, second and third molars, and the distances were measured using built-in measurement tools. Images were grouped according to the relation between the root tips and the maxillary sinus floor, as follows: Group 1: Root tips in contact with the sinus floor (Figure 1); Root tips penetrating into the sinus (Figure 2); and Group 3: Root tips below the sinus floor (Figure 3). Distances were measured for each side of each tooth and root tip. Root tips in Group 1 were numbered as zero, those in
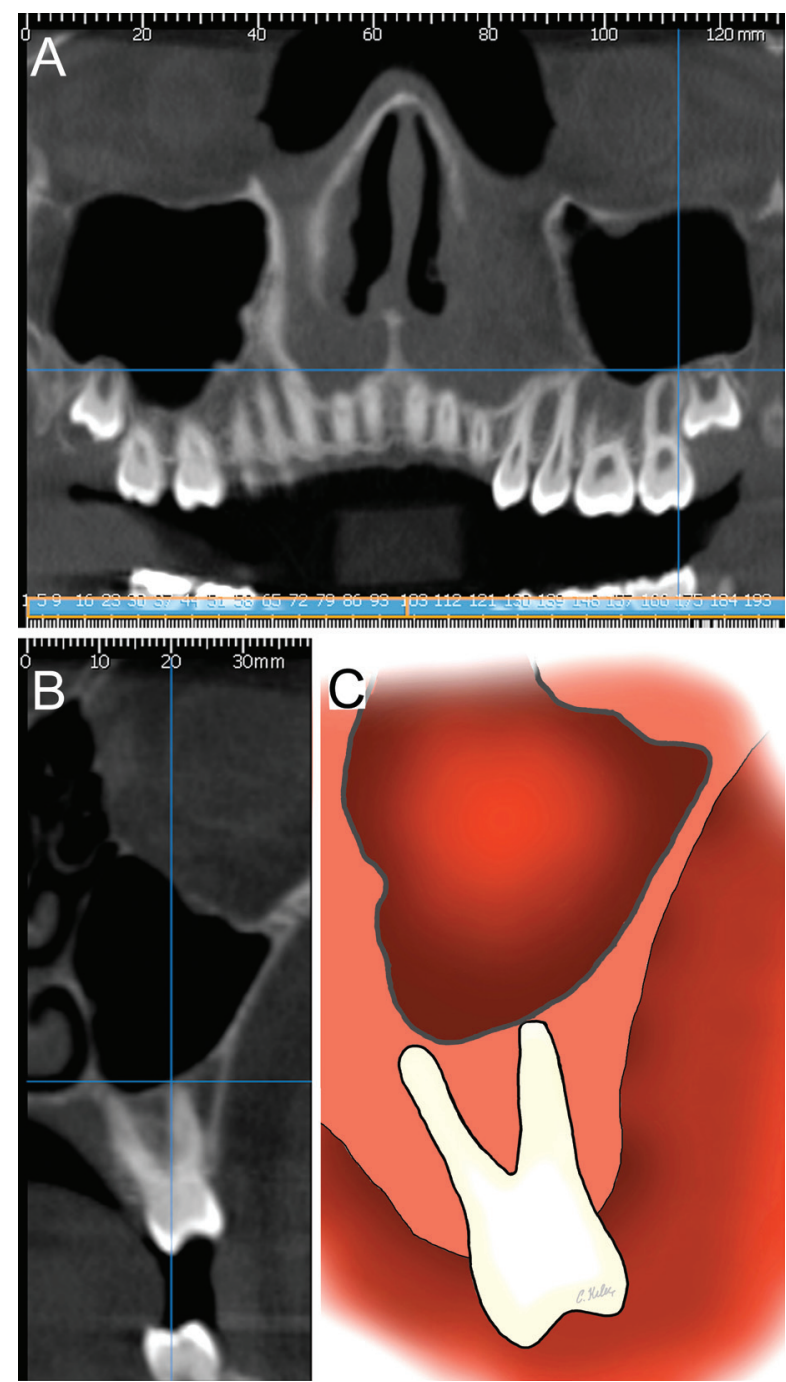

Figure 1. A: Tangential view of left second molar tooth. B: Anterior view of the tooth C: The schematic drawing of the image $B$. 
Group 2 were given negative numbers and those in Group 3 were given positive numbers. Means, standard deviations and minimum and maximum values were calculated for all right and left premolars and molars. T-tests were used to compare measurements between left and right sides and between female and male patients.

\section{RESULTS}

Means, standard deviations and minimum and maximum values obtained from right and left premolars and molars are given in Tables 1 and 2 . The distance between sinus floor and root tip was longest for the first premolar root tip and shortest for the second molar buccodistal root tip for both right and left sides. No statistically significant differences were found between the measurements for right and left sides ( $P>$.05) or between female and male patients $(P>.05)$. Totally, for the right side $60 \%$ of the root tips were included in group
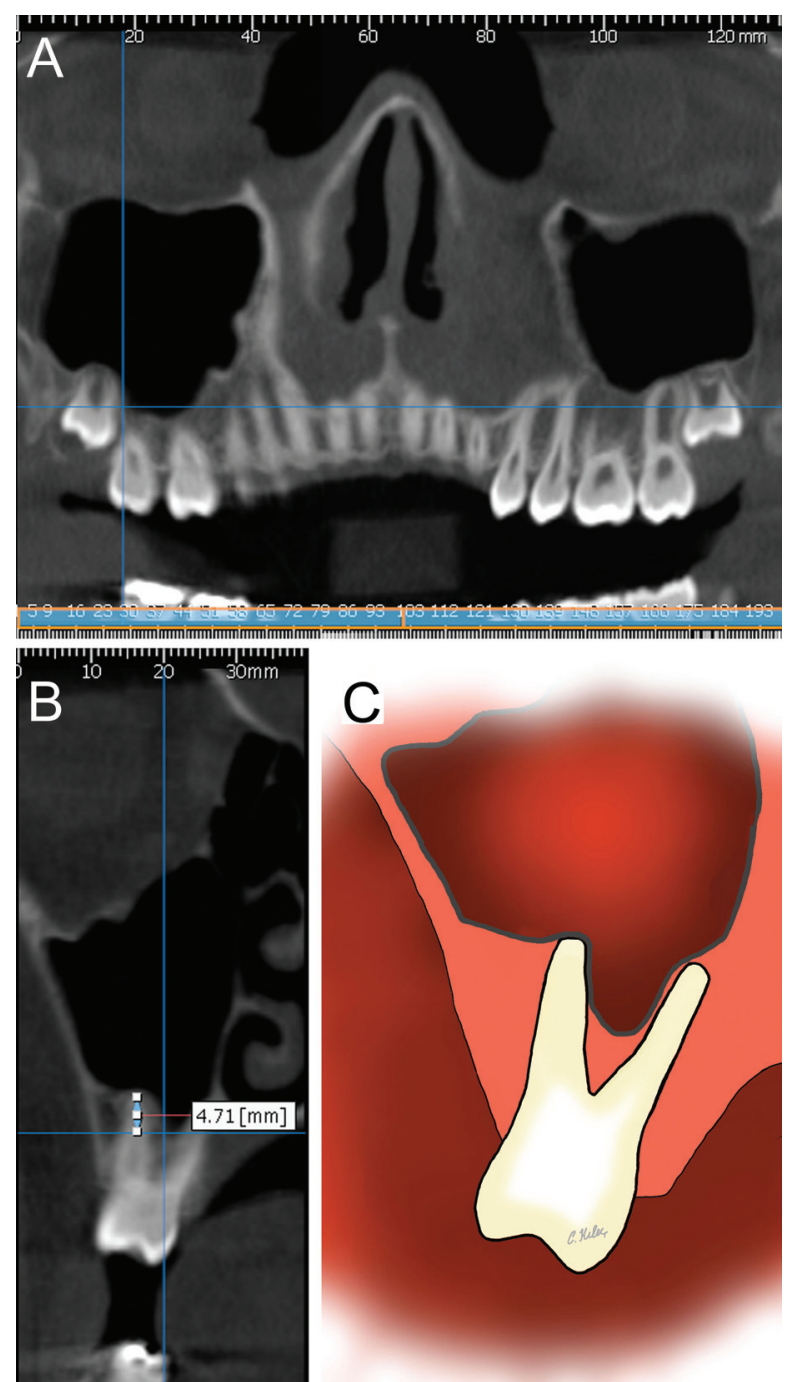

Figure 2. A: Tangential view of right second molar tooth. B: Anterior view of the tooth. C: The schematic drawing of the image B.
$3,30 \%$ in group 1 and $10 \%$ in group 2 whereas on the left side $68 \%$ were included in group $3,21 \%$ in group 1 and $11 \%$ in group 2 .

\section{DISCUSSION}

Our study documents the anatomical relationship between the root tips of the maxillary posterior teeth and the maxillary sinus floor. Both left and right sides of each patient were evaluated separately.

Clinicians conducting preprosthetic and preimplant surgical procedures in the posterior maxilla must be aware of the degree to which teeth roots protrude into the sinus because of the associated risk of post-extraction pneumatization, ${ }^{6}$ which reduces the amount of bone available at the implant or denture site. In most cases, only a panoramic radiograph is available to the clinician, and only a few clinicians order a cone-beam CT. ${ }^{12}$ In one study that aimed to compare the relationship of maxillary teeth roots to the bottom of the maxillary sinus, panoramic radiographs and CTs of 30 patients were examined. The topographic relationships between the roots and sinus as measured using panoramic radiographs were significantly different from those measured from CTs, which were taken an average of 2.5 months after
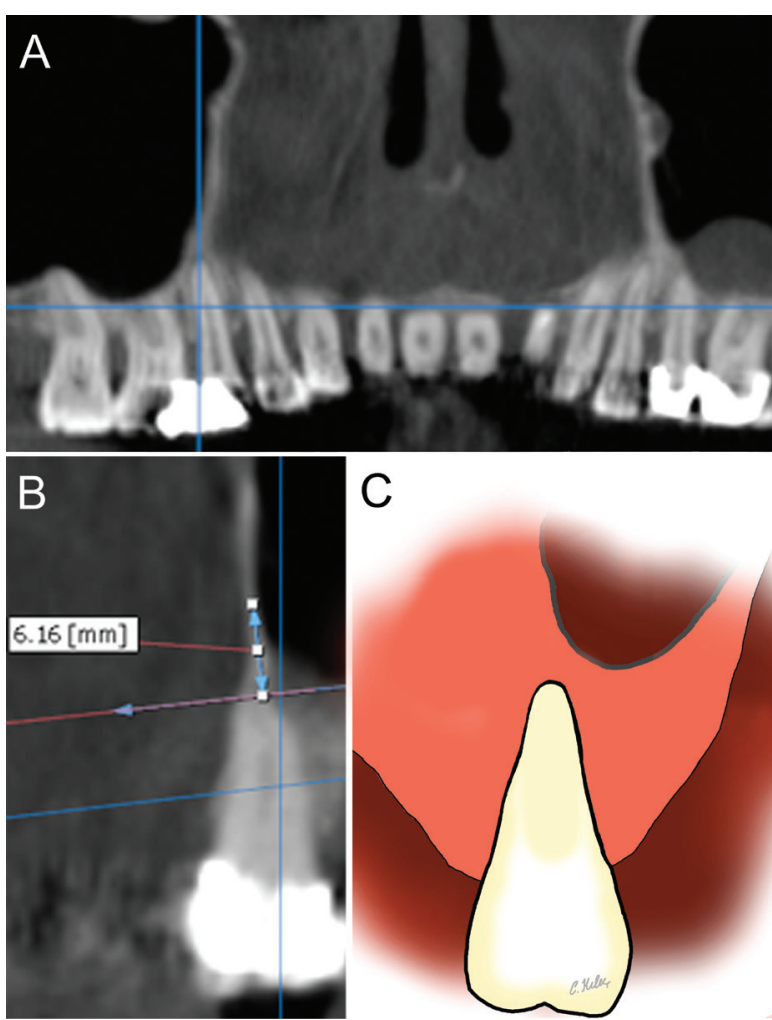

Figure 3. A: Tangential view of right second premolar tooth. B: Anterior view of the tooth. C: The schematic drawing of the image B. 
the radiographs. In the panoramic radiographs, 64 out of 129 roots appeared to penetrate the maxillary sinus, as compared to 37 of 129 roots in the CT transversal slices. ${ }^{13}$ In order to avoid disadvantages such as superposition of anatomic structures, horizontal and vertical magnification and a lack of cross-sectional information that are associated with panoramic radiographs, the present study was conducted using cone-beam CT images only.

In a study that measured mean distances between maxillary posterior teeth apices and the maxillary sinus floor and between the apices and the adjacent lateral bony surfaces using CT display data from 12 autopsy specimens and $38 \mathrm{hu}-$ man subjects, the apex of the maxillary second molar mesiobuccal root was found to be closest to the sinus floor (mean: $1.97 \mathrm{~mm}$ ) and farthest from the buccal bony surface (mean: $4.45 \mathrm{~mm}$ ), whereas the apex of the maxillary first premolar buccal root was found to be closest to the adjacent lateral bony surface (mean: $1.63 \mathrm{~mm}$ ) and farthest from the sinus floor (mean: $7.05 \mathrm{~mm}$ ). ${ }^{14}$ The present study also found the first premolar root tip to be farthest and the second molar buccodistal root tip to be closest to the sinus floor on both right and left sides. Another study by Kwak et $\mathrm{al}^{15}$ that used CT images and described 5 vertical relationships and 3 horizontal relationships found the most frequent vertical relationship to be one in which teeth roots had no contact with the sinus floor. Similarly, in the present study the most frequent vertical relationship was found to be one in which

Table 1. Mean, standard deviation, minimum and maximum values obtained from right premolar and molar teeth.

\begin{tabular}{|c|c|c|c|c|c|}
\hline & $\mathrm{N}$ & Mean & Std. Dev. & Minimum & Maximum \\
\hline R 1st mo bm & 87 & 1.77 & 6.10 & -5.41 & 27.55 \\
\hline R 1 st mo pal & 87 & 1.86 & 6.06 & -4.22 & 29.46 \\
\hline R 2nd mo bm & 87 & 0.42 & 2.85 & -5.06 & 16.45 \\
\hline $\mathrm{R}$ 2nd mo bd & 87 & 0.25 & 2.17 & -5.97 & 8.76 \\
\hline R 2nd mo pal & 87 & 1.06 & 2.36 & -4.52 & 9.57 \\
\hline R 3rd mo pal & 87 & 0.92 & 3.32 & -2.87 & 8.54 \\
\hline
\end{tabular}

R- right, pm- premolar, mo- molar, bm-buccomesial, bd-buccodistal, pal-palatinal

Table 2. Mean, standard deviation, minimum and maximum values obtained from left premolar and molar teeth

\begin{tabular}{|c|c|c|c|c|c|}
\hline L 1st pm & 89 & 6.58 & 8.61 & -1.88 & 25.26 \\
\hline L 1st mo bm & 89 & 0.52 & 2.80 & -5.13 & 15.20 \\
\hline L 1st mo pal & 89 & 0.26 & 0.69 & -0.24 & 2.74 \\
\hline L 2nd mo bm & 89 & 0.31 & 3.13 & -8.67 & 14.98 \\
\hline L 2nd mo bd & 89 & 0.40 & 2.50 & -8.93 & 9.90 \\
\hline L 3rd mo pal & 89 & 0.94 & 1.79 & -0.82 & 4.77 \\
\hline
\end{tabular}

L- Left, pm- premolar, mo- molar, bm-buccomesial, bd-buccodistal, pal-palatinal 
roots had no contact with sinus floor for both right and left sides.

Because of the very close anatomical relationship that exists between the maxillary posterior teeth root tips and the sinus floor, endodontic surgery of premolars and molars can result in accidental oroantral communication ${ }^{16-18}$ that can allow bacteria from infected periapical tissue, resected root tips, or bony drilling dust to be displaced into the sinus and cause acute or chronic sinusitis. ${ }^{18}$ Surgical treatment of posterior teeth is also complicated by the restricted space of the oral vestibular region, which in turn makes it difficult to raise a flap. ${ }^{19}$

Various authors have studied the relationship between the maxillary molar and premolar roots and the maxillary sinus. ${ }^{3,4,14,20}$ Eberhardt et $\mathrm{al}^{14}$ found the mean distance between the maxillary posterior teeth and the maxillary sinus floor to be $1.97 \mathrm{~mm}$. The roots of the maxillary first and second molars have been shown to be in an intimate relationship with the maxillary sinus floor in 40 percent of cases. ${ }^{20}$ The palatine roots have been shown to be closer to the antral floor than to the palate and in close proximity to the maxillary sinus in 20 percent of cases. ${ }^{4}$ This location complicates a surgical approach through the sinus, so that palatinal access is usually required. ${ }^{14}$ Although the vestibular roots of the upper posterior teeth are also in close contact with the maxillary sinus floor, vestibular roots are much easier to access than pa-latinal roots, so that treatment can usually be carried out without perforating the sinus wall. ${ }^{3}$ In some cases, the root apices protrude into the sinus so that treatment requires raising the sinus membrane. ${ }^{21}$

As with other apicoectomies, complications encountered during periapical surgery of the maxillary molars and premolars can include damage to a neighboring tooth. With regard to the specific treatment of maxillary molars and premolars, careful aperture of the maxillary sinus wall or floor is necessary, and attention must be paid to avoid sinus membrane perforation and the introduction of foreign bodies into the maxillary sinus. ${ }^{18,20,22,23}$ Ericson et $\mathrm{al}^{6}$ reported that out of 159 maxillary premolars and molars treated with periapical surgery, aperture of the wall or floor of the maxillary sinus occurred in 18 percent of cases. The authors also stated that the introduction of foreign bodies into the maxillary sinus during surgery could cause thickening of the sinus mucosa and symptoms of maxillary sinusitis. In order to avoid penetration by foreign bodies, Jerome and Hill ${ }^{24}$ recommend using gauze to block the maxillary sinus aperture. Friedman et a ${ }^{25}$ performed periapical surgery on 94 maxillary teeth roots, 12 of which were maxillary molar roots, and reported an 11.8 percent rate of aperture of the sinus wall or floor. According to Selden, ${ }^{26}$ pathological exposure of the maxillary sinus floor during periapical surgery predisposes to orosinusal communications.

Regarding sinus membrane perforation, Persson $^{23}$ performed periapical surgery on 18 maxillary molars, with a perforation rate of 44 percent. Despite this complication, the reported surgical success rate was 78 percent, and no relationship was observed between membrane perforation and surgical outcome. loannides and Borstlap ${ }^{17}$ performed surgery on 47 maxillary molars, with a perforation rate of 14.8 percent. According to these authors, perforation of the membrane did not affect the formation of periapical bone.

\section{CONCLUSIONS}

Knowledge of the anatomical relationship between the maxillary sinus floor and the maxillary posterior teeth root tips is important for the preoperative treatment planning of maxillary posterior teeth. In view of the proximity of the maxillary sinus floor and maxillary root tips, clinicians must be particularly cautious when performing dental procedures involving the maxillary posterior teeth. The measurements found in the present study highlight the need for preoperative treatment planning.

\section{ACKNOWLEDGEMENTS}

The authors would like to thank Dr. A.R. Ilker Cebeci for providing the ILUMA unit and Deborah Semel for her editorial assistance.

\section{REFERENCES}

1. Misch CE. Contemporary implant dentistry. 2nd ed. St.Louis: CV Mosby Co, 1999:76-194.

2. Mc Growan DA, Baxter PW, James J. The Maxillary Sinus and its Dental Implications. 1st ed. London: Wright Co., 1993:1-25 
3. Hauman $\mathrm{CH}$, Chandler NP, Tong DC. Endodontic implications of the maxillary sinus: a review. Int Endod $J$ 2002;35:127-141.

4. Waite DE. Maxillary sinus. Dent Clin North Am 1971;15:349368.

5. Tank PW. Grant's Dissector. 13 ed. Philadelphia: Lippincott Williams \& Wilkins, 2005:198.

6. Wehrbein $H$, Diedrich P. The initial morphological state in the basally pneumatized maxillary sinus-a radiological-histological study in man. Fortschr Kieferorthop 1992;53:254-262.

7. Engstrom H, Chamberlain D, Kiger R, Egelberg J. Radiographic evaluation of the effect of initial periodontal therapy on thickness of the maxillary sinus mucosa. J Periodontol 1988;59:604-698.

8. Monkhouse S. Cranial Nerves Functional Anatomy. 2nd ed. New York: Cambridge University Press, 2006:59.

9. Williams PL, Bannister LH, Berry MM et al. Gray's anatomy. 38th ed. New York: Churchill-Livingstone, 1995:1239.

10. Harrison DF. Oro-antral fistula. Br J Clin Pract 1961;15:169174.

11. Fuhrmann R, Bucker A, Diedrich. Radiological assessment of artificial bone defects in the floor of the maxillary sinus. Dentomaxillofac Radiol 1997;26:112-116.

12. Arbel Sharan, David Madjar. Correlation between maxillary sinus floor topography and related root position of posterior teeth using panoramic and cross-sectional computed tomography imaging. Oral Surg Oral Med Oral Pathol Oral Radiol Endod 2006;102:375-381.

13. Freisfeld M, Drescher D, Schellmann B, Schüller $H$. The maxillary sixth-year molar and its relation to the maxillary sinus. A comparative study between the panoramic tomogram and the computed tomogram. Fortschr Kieferorthop 1993;54:179-186.

14. Eberhardt JA, Torabinejad M, Christiansen EL. A computed tomographic study of the distances between the maxillary sinus floor and the apices of the maxillary posterior teeth. Oral Surg Oral Med Oral Pathol 1992;73:345-346.

15. Kwak HH, Park HD, Yoon HR, Kang MK, Koh KS, Kim HJ. Topographic anatomy of the inferior wall of the maxillary sinus in Koreans. Int J Oral Maxillofac Surg 2004;33:382388.

16. Ericson S, Finne K, Persson G. Results of apicoectomy of maxillary canines, premolars and molars with special reference to oroantral commu-nication as a prognostic factor. Int J Oral Surg 1974;3:386-393.

17. Ioannides C, Borstlap WA. Apicoectomy on molars: a clinical and radiographical study. Int J Oral Surg 1983;12:73-79.
18. Watzek G, Bernhart T, Ulm C. Complications of sinus perforations and their management in endodontics. Dent Clin North Am 1997;41:563-583.

19. Gay C, Paredes J, Berini L. La cirugía periapical en los molares. Rev Eur Odont Estomatol 1993;2:95-102.

20. Wallace JA. Transantral endodontic surgery. Oral Surg Oral Med Oral Pathol 1996;82:80-83.

21. Berta García, Luis Martorell, Eva Martí, Miguel Peñarrocha. Periapical surgery of maxillary posterior teeth. A review of the literature. Med Oral Patol Oral Cir Bucal 2006;11:E146E150.

22. Khongkhunthian P, Reichart PA. Aspergillosis of the maxillary sinus as a complication of overfilling root canal material into the sinus: report of two cases. $J$ Endod 2001;27:476-478.

23. Persson G. Periapical surgery of molars. Int J Oral Surg 1982;11:96-100.

24. Jerome CE, Hill AV. Preventing root tip loss in the maxillary sinus during endodontic surgery. J Endod 1995;21:422-424.

25. Friedman S, Lustmann J, Shaharabany V. Treatment results of apical surgery in premolar and molar teeth. $J$ Endod 1991;17:30-33.

26. Selden HS. The interrelationship between the maxillary sinus and endodontics. Oral Surg Oral Med Oral Pathol 1974;38:623-629. 